\title{
A comparative study of modelling AC electric arc by one-dimensional interpolation for power system harmonics analysis
}

\author{
Yu-Jen Liu, Jen-Pan Hung, Shang-I Chen and Cheng-Wei Lin \\ National Chung Cheng University, Department of Electrical Engineering, 62102 Chia-Yi, Taiwan (R.O.C.)
}

\begin{abstract}
Electric arc is a discharge phenomenon caused by particular electrical events and arc produced facilities in power system, for example the occurrence of short-circuit fault in feeders and the use of electric arc furnace for steelmaking. All of these electric arcs have a highly nonlinear nature and can be considered as a significant source of power quality problems. To investigate the impacts of the electric arcs on power quality studies the development of the electric arc models for simulation analysis is became necessary. This paper thus presents the use of different onedimensional interpolation methods for modelling the alternating current electric arc. The performances of different methods are compared by simulation results that are derived from the modelling of an alternating current electric arc furnace load and a low-frequency fluorescent tube.
\end{abstract}

\section{Introduction}

It is known that computer-aided simulation analysis is one of the most effective ways to assess the power quality (PQ) problems in power system. Therefore, to assist the simulation of the operation of an electric power network controlling an electric arc load, for instance an arcdischarge fluorescence lamp/tube (FL/FT) or an electric arc furnace (EAF), an appropriate model for the electric arc load is necessary. The usefulness of simulation performance highly relies on the applicable models of the electric components that are used to describe the operation of the system and a well understanding of the sensitivities of the models with respect to the data available for model construction. Due to the complex electric arc property, the $\mathrm{v}-\mathrm{i}$ characteristic of the arc voltage and current is always nonlinear and it is thus difficult to develop a deterministic electric arc model for study.

In past efforts, many approaches are provided for electric arc modelling. For example, references [1-3] model the electric arc by a resistance and nonlinear resistance but they cannot suitably present the physics behaviour of the electric arc. Electric arc conductance models are proposed by $[4,5]$ that are classic differential equations for representation of an arc with high or low current conditions, separately. Harmonic voltage model and harmonic current injection model are the two common used methods for electric arc modelling in power quality study that are based on the field measurements of arc voltage and current [6]. In addition, some intelligent methods such as artificial neural network and wavelet transform have been proposed for specified studies and the performance of them are proved to be effective; however, the structures of these models are more complicated and thus is not easily be carried out on simulation. To provide an efficient and easy work modelling approach for the electric arc on PQ study, especially for the harmonic problem analysis, this paper presents the comparative study of using one-dimensional interpolation methods for electric arc modelling. Measured electric arc data that are collected from an alternating current (AC) EAF and a traditional T9 lowfrequency FT are used for simulations and model validations. The performance of different interpolation models are then compared by simulation results for the assessments of harmonic distortions on the AC EAF and FT. The organization of the paper is as follows. In Section II, a brief description of the characteristic of electric arc is presented. The concept of modeling AC electric arc with different interpolation methods is addressed in Section III. Experiments and simulation results are illustrated in Section IV to show the performance of the methods for modeling the AC electric arc, and then the conclusion follows.

\section{Characteristics of the $\mathrm{AC}$ electric arc}

This paper mainly discusses the modelling issue of $\mathrm{AC}$ electric arc which is a gas ionization phenomenon that produces continued electrical discharge between two electrodes. An AC electric arc may re-strike on each half cycle of the fundamental frequency current period. The common features of the AC electric arc can be characterized by time-domain waveforms and the distributions of frequency spectra in frequency-domain [7]. Figure 1 shows the measured AC electric arc signal; from the view of observing time-domain waveform, all 
AC electric arcs consist of three regions that named cathode drop zone, anode drop zone and arc column no matter what the total arc length is. The major arc voltage is developed within the arc column, and the cathode drop (Vc) and anode drop (Va) of a few volts are presented within a little bit millimetre of each electrode. The arc voltage will reach to peak value (reignited voltage), after the zero-crossing point, when the current lies in near zero duration (shoulders phenomenon), [8], and the shape of the arc voltage waveform is closed to square-wave. To compare with a pure sinusoidal current waveform, the peak value of the arc current may less than it and has a steep current raise rate when the current increase. Furthermore, the arc voltage and arc current are sometimes in phase, which hence presents the resistive/conductance properties on its $\mathrm{v}-\mathrm{i}$ characteristic.

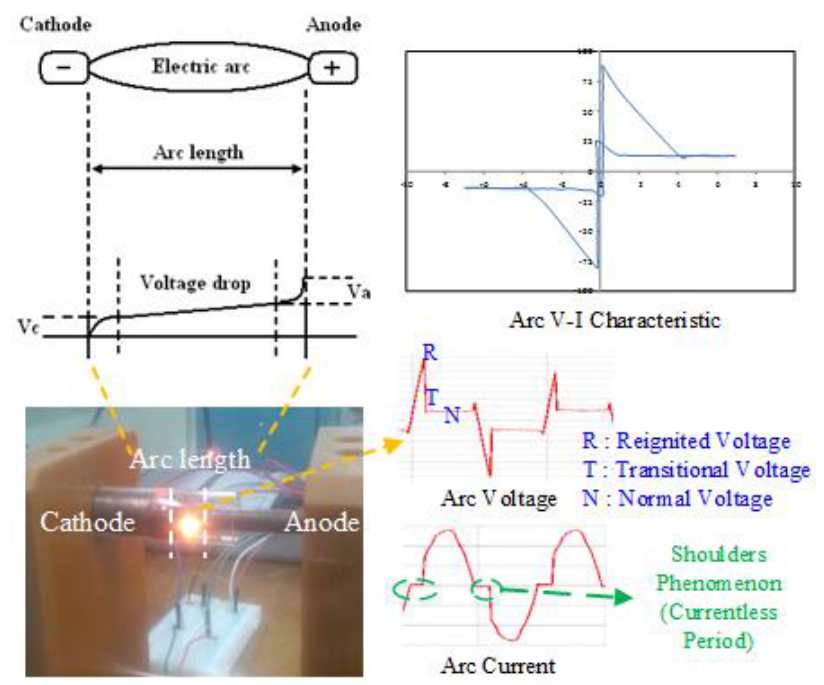

Figure 1. Characteristics of the AC electric arc.

\section{Mathematical modelling}

In practice, the field measurement of $\mathrm{AC}$ electric arc voltages and currents is the most effective way to obtain the required electrical parameters for modelling purpose. The modelling concept in this study is to develop the nonlinear conductance model based on different interpolation techniques; meanwhile, the parameters of AC electric arc conductance are collected from the above-mentioned field measurement. Interpolation is used to estimate new arc conductance values between consecutive known data points from measurements. Figure 2 shows the diagram of the presented models in this paper and the descriptions of each modelling method are illustrated as following:

\subsection{Nearest neighbor interpolation}

Nearest neighbor is the most basic interpolation technique and requires the least processing time of all the interpolation algorithms. This method sets the value of an interpolated point to the value of the nearest data point. Therefore, this method does not generate any new data points. Equation (1) shows the arc conductance interpolant function of the method; meanwhile, $x$ is the original point closest to $x$ '. Figure 3 presents the concept of this method.

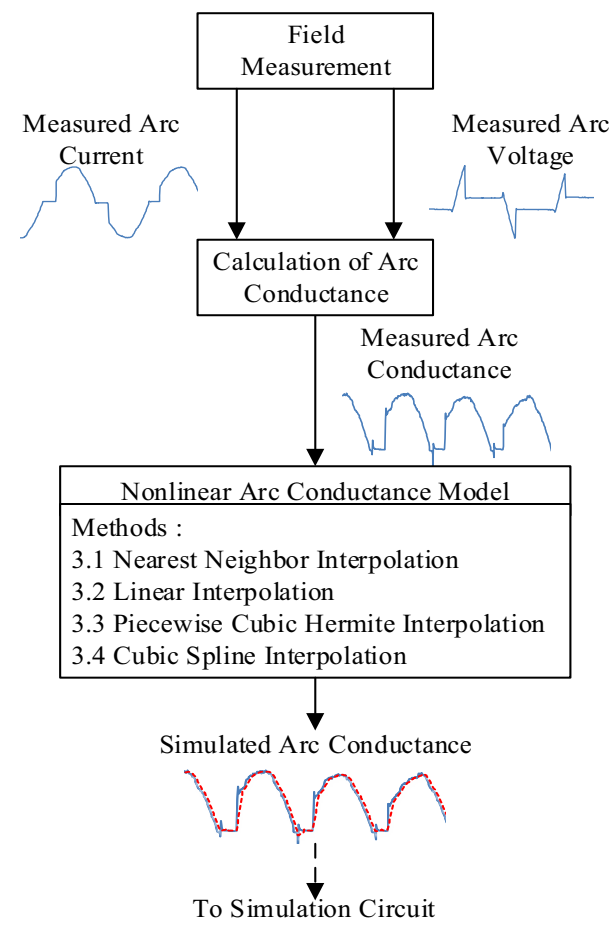

Figure 2. The concept of modelling AC electric arc by interpolation methods.

$$
G\left(x^{\prime}\right)=f(x)
$$

- Original point

○ Interpolated value

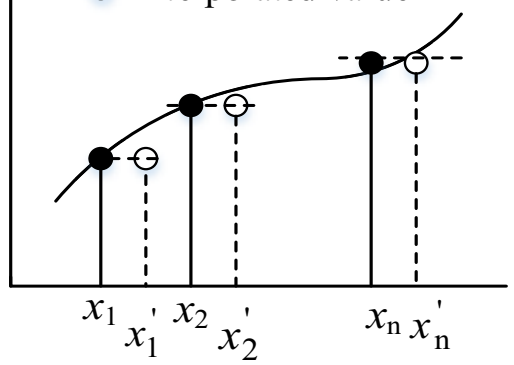

Figure 3. Nearest neighbor interpolation.

\subsection{Linear interpolation}

The linear interpolation is piecewise straight lines connecting two consecutive data points. The arc conductance linear interpolant function in the interval $\left[x_{\mathrm{i}}\right.$, $\left.x_{\mathrm{i}+1}\right]$ is given

$$
G(x)=y_{\mathrm{i}}+s \cdot \delta_{\mathrm{i}}
$$

It must pass through $\left(x_{\mathrm{i}}, y_{\mathrm{i}}\right)$ and $\left(x_{\mathrm{i}+1}, y_{\mathrm{i}+1}\right)$ and a continuity condition of $G_{\mathrm{i}}\left(x_{\mathrm{i}+1}\right)=G_{\mathrm{i}+1}\left(x_{\mathrm{i}+1}\right)$ must be satisfied. In (2), $y$ is arc conductance at original data point i, $\delta_{\mathrm{i}}=\left(y_{\mathrm{i}+1}-y_{\mathrm{i}}\right) /\left(x_{\mathrm{i}+1}-x_{\mathrm{i}}\right)$ presents the slope of the line and the local variable $s=x-x_{\mathrm{i}}$.

\subsection{Piecewise cubic Hermite interpolation (PCHIP)}


This interpolation method is to develop an interpolant cubic polynomial of the arc conductance function at known data points among a whole interval $\left[x_{0}, x_{\mathrm{n}}\right]$. To this end, the general cubic polynomial at $\mathrm{i}^{\text {th }}$ interval is defined by

$$
P_{\mathrm{i}}(x)=a_{\mathrm{i}}+b_{\mathrm{i}}\left(x-x_{\mathrm{i}}\right)+c_{\mathrm{i}}\left(x-x_{\mathrm{i}}\right)^{2}+d_{\mathrm{i}}\left(x-x_{\mathrm{i}}\right)^{3}
$$

where $P$ is arc conductance; $a, b$, c, and $d$ are coefficients to be found; $\mathrm{i}=0,1,2, \ldots, \mathrm{n}$; and the completed arc conductance model by PCHIP is given by

$$
G(x)=\left\{\begin{array}{cc}
P_{0}(x) & x_{0} \leq x \leq x_{1} \\
P_{1}(x) & x_{1} \leq x \leq x_{2} \\
\vdots & \vdots \\
P_{\mathrm{n}}(x) & x_{\mathrm{n}-1} \leq x \leq x_{\mathrm{n}}
\end{array}\right.
$$

For a function $P$ in (3), in order to find the coefficients of $a$ to $d$ it must satisfies four interpolation conditions, two on function values and two on the possibly unknown derivative values, which are given in (5) to (8).

$$
\begin{aligned}
& P_{\mathrm{i}}\left(x_{\mathrm{i}}\right)=f\left(x_{\mathrm{i}}\right) \\
& P_{\mathrm{i}}\left(x_{\mathrm{i}+1}\right)=f\left(x_{\mathrm{i}+1}\right) \\
& P_{\mathrm{i}+1}\left(x_{\mathrm{i}+1}\right)=P_{\mathrm{i}}\left(x_{\mathrm{i}+1}\right) \\
& P_{\mathrm{i}+1}^{\prime}\left(x_{\mathrm{i}+1}\right)=P_{\mathrm{i}}^{\prime}\left(x_{\mathrm{i}+1}\right)
\end{aligned}
$$

If both function values and first derivative values at a set of data points can be known then PCHIP can reproduce those data.

\subsection{Cubic spline interpolation}

The method is also used to create a similar cubic polynomial as in (3) but that is continuous in both the first and second derivatives. Therefore, besides (5) to (8) an additional condition in (9) must be satisfied as well.

$$
P_{\mathrm{i}+1}^{\prime \prime}\left(x_{\mathrm{i}+1}\right)=P_{\mathrm{i}}^{\prime \prime}\left(x_{\mathrm{i}+1}\right)
$$

For a unique solution, it needs to specify further end (or boundary) conditions at $x_{0}$ and $x_{\mathrm{n}}$, and the most common used are

- Natural end condition - assume the second derivative at the end points are zero

$$
P^{\prime \prime}\left(x_{0}\right)=P^{\prime \prime}\left(x_{\mathrm{n}}\right)=0
$$

- Clamped end condition - assume the first derivatives at the first and last points are known

$$
P^{\prime}\left(x_{0}\right)=f^{\prime}\left(x_{0}\right) ; P^{\prime}\left(x_{\mathrm{n}}\right)=f^{\prime}\left(x_{\mathrm{n}}\right)
$$

- Not-a-knot end condition - force continuity of the third derivative at the second and penultimate points, which result in the first two intervals having the same spline function and the last two intervals having the same spline function. In this paper, only this end condition is considered for modelling.

$$
P^{\prime \prime \prime}\left(x_{0}\right)=f^{\prime \prime \prime}\left(x_{1}\right) ; P^{\prime \prime \prime}\left(x_{\mathrm{n}}\right)=f^{\prime \prime \prime}\left(x_{\mathrm{n}-1}\right)
$$

\section{Experiments and simulations}

Two case studies are presented to estimate the performance of the different models:

\subsection{AC electric arc furnace modelling}

Figure 4 shows the one-line diagram of the measured steel plant power system which includes a 50 ton AC EAF load. For harmonic distortion analysis, the EAF voltages and currents are recorded at the duration when the EAF load operates at refine stage (static-state condition). Measured EAF characteristics are given in Fig. 5 and simulated results for each interpolation method are shown in Fig. 6 to Fig. 9. To observe the time-domain waveforms in these results, some of the features are found: (i) higher odd harmonic components in voltage and current are provided by the AC EAF load; (ii) the non-smooth serrated waveforms are clearly obtained by Nearest; (iii) arc voltage waveform is distorted in Linear; and (iv) the results from PCHIP and Spline are similar but the differences are presented in data boundary. Figure 10 shows the harmonic spectra of EAF voltage and current, and the total harmonic voltage/current distortions $\left(\mathrm{THD}_{\mathrm{V}} / \mathrm{THD}_{\mathrm{I}}\right)$ relate with these spectra are listed in Table 1. It is found the better performance can be presented by using Spline which provides about 3.98\% and 3.09 errors on $\mathrm{THD}_{\mathrm{V}}$ and $\mathrm{THD}_{\mathrm{I}}$, separately.

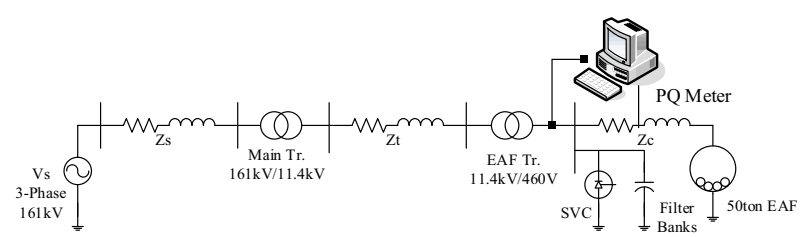

Figure 4. One-line diagram of the measured steel plant power system.

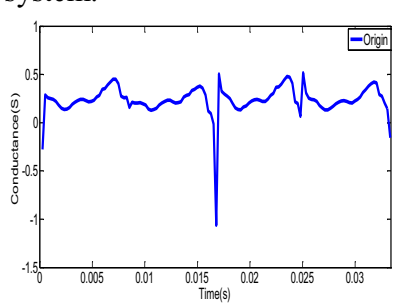

(a) Conductance



(c) EAF voltage

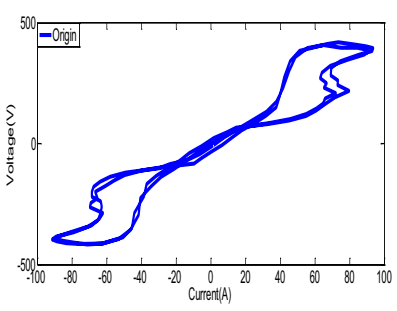

(b) v-i characteristic

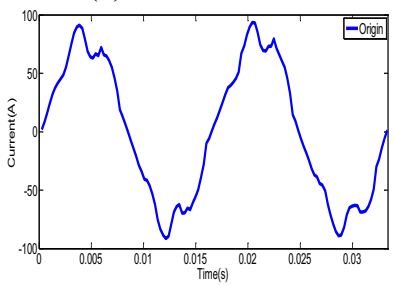

(d) EAF current
Figure 5. Measured results. 


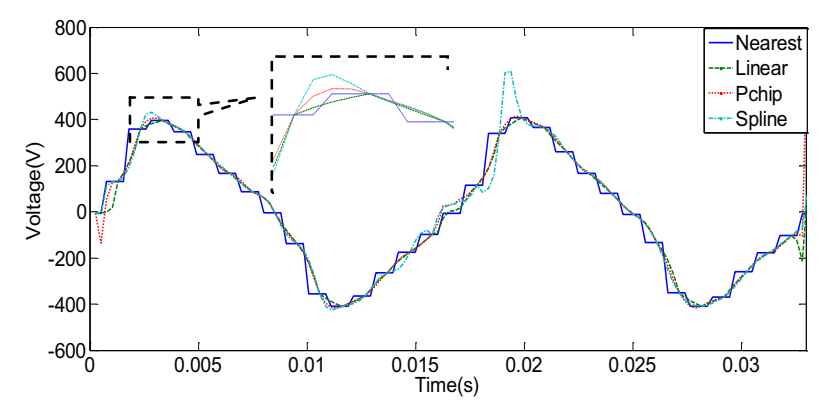

Figure 6. Simulated conductance.

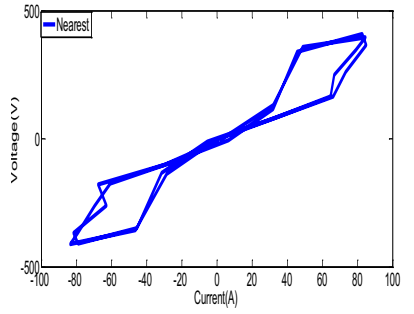

(a) Nearest

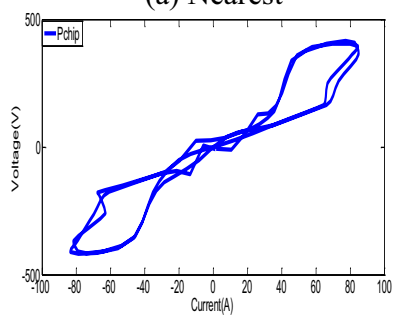

(c) Pchip

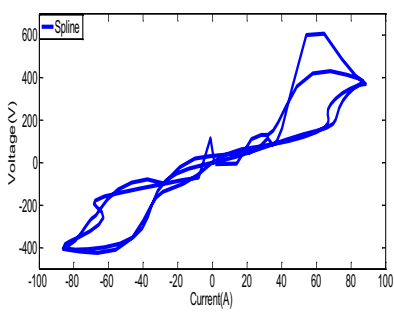

(b) Linear

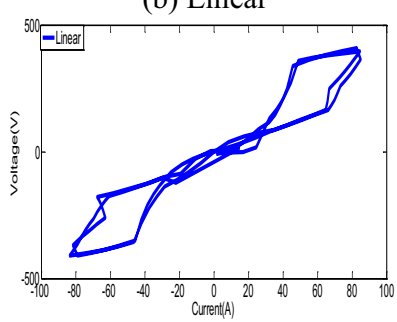

(d) Spline
Figure 7. Simulated v-i characteristic.

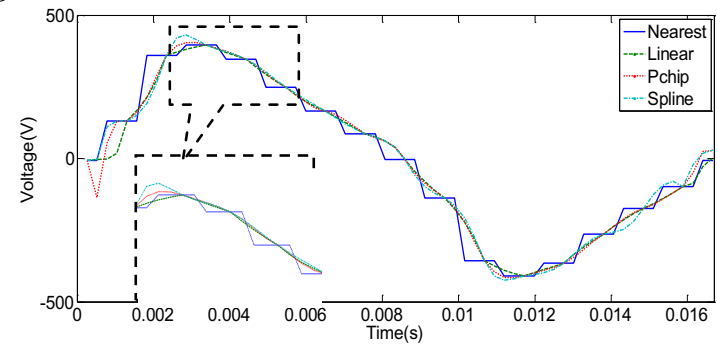

Figure 8. Simulated EAF voltage.

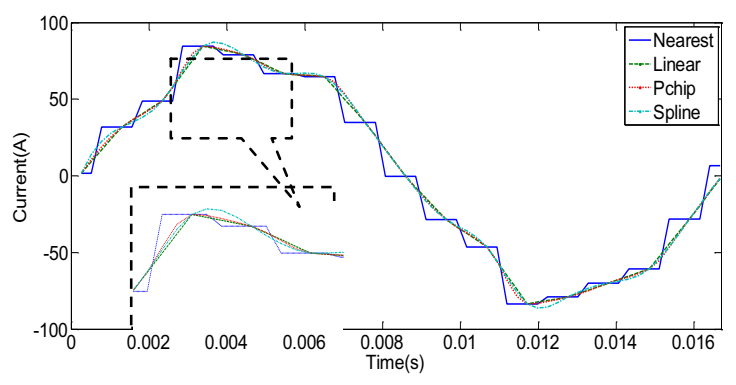

Figure 9. Simulated EAF current.

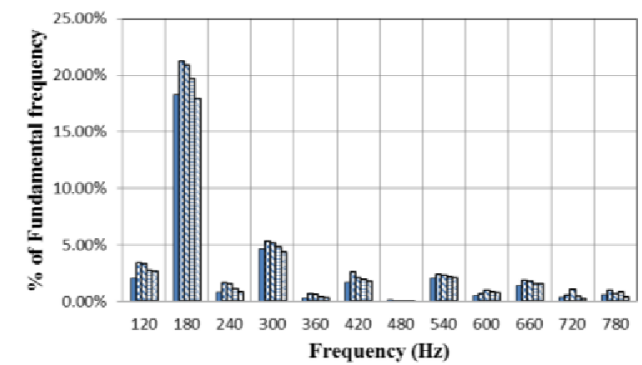

(a) Voltage

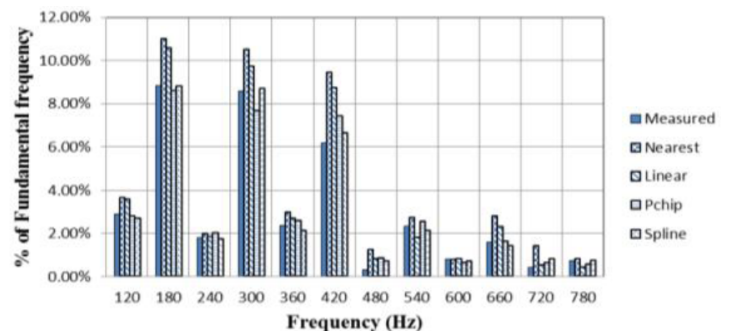

(b) Current

Figure 10. Harmonic spectra of EAF.

Table 1. Total harmonic distortion of EAF voltages and currents.

\begin{tabular}{|c|c|c|}
\hline Method & THD $_{\mathbf{V}} \mathbf{( \% )}$ & THD $_{\mathbf{I}} \mathbf{( \% )}$ \\
\hline Measured & 19.25 & 13.44 \\
\hline Nearest & 29.44 & 23.92 \\
\hline Linear & 27.72 & 20.28 \\
\hline Pchip & 24.47 & 18.46 \\
\hline Spline & 23.21 & 16.53 \\
\hline
\end{tabular}

\subsection{Low frequency fluorescent tube modelling}

Fluorescent lighting generally requires a relatively long evacuated tube to develop sufficient ionized gas for operation. It is possible to have a nonlinear nature in the $\mathrm{v}-\mathrm{i}$ characteristic and thus the tube current for an applied sinusoidal voltage is rich in odd harmonics. This can be observed from the test circuit in Fig. 11 and measured results in Fig. 12. To observe the simulation results on Fig. 13 to Fig. 17 it is found that the arc conductance on fluorescent tube presents a regular variation than EAF load, and harmonics distortions shown in Table 2 present smaller differences among each method when compared to measured results.

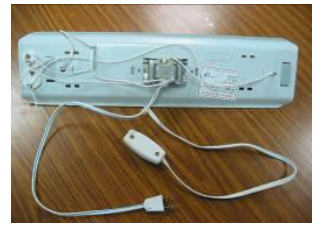

(a)

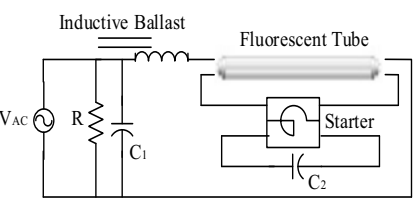

(b)
Figure 11. Fluorescent tube circuit.



(a) Conductance

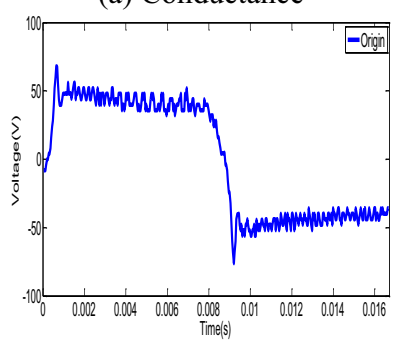

(c) Arc voltage

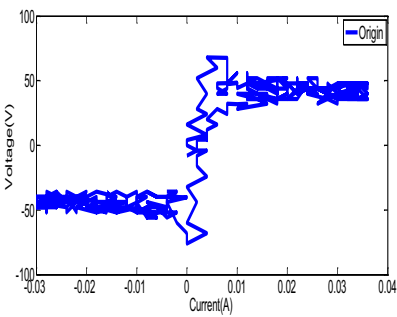

(b) v-i characteristic

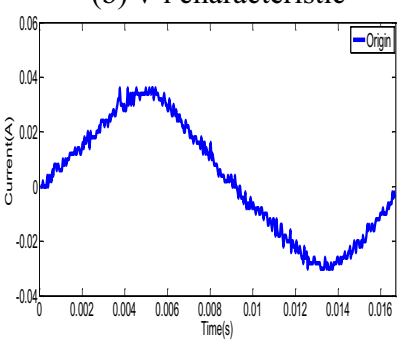

(d) Arc current
Figure 12. Measured results. 




Figure 13. Simulated conductance.



(a) Nearest

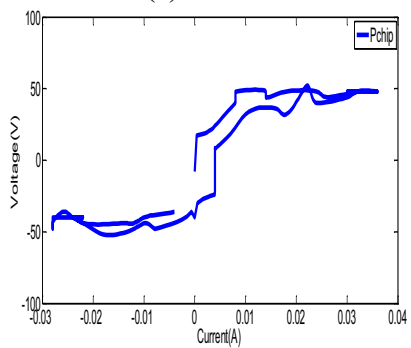

(c) Pchip

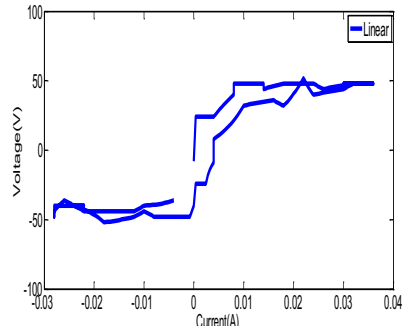

(b) Linear

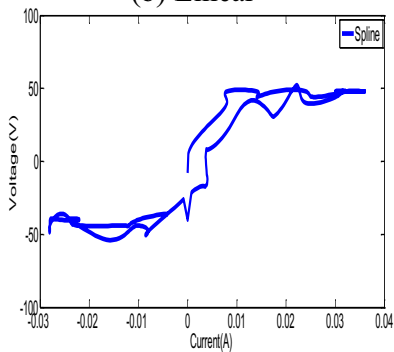

(d) Spline
Figure 14. Simulated v-i characteristic.



Figure 15. Simulated FT voltage.

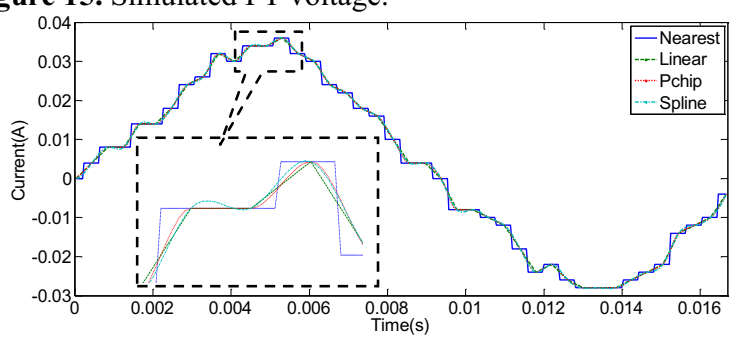

Figure 16. Simulated FT current.



(a) Voltage

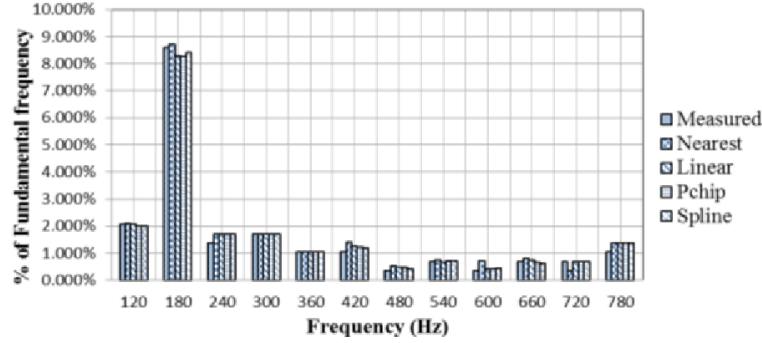

(b) Current

Figure 17. Harmonic Spectra of FT.

Table 2. Total harmonic distortion of lamp voltages and currents

\begin{tabular}{|c|c|c|}
\hline Method Index & THD $_{\mathbf{V}}(\mathbf{\%})$ & THD $_{\mathbf{I}} \mathbf{( \% )}$ \\
\hline Measured & 40.16 & 11.71 \\
\hline Nearest & 36.61 & 12.44 \\
\hline Linear & 36.36 & 10.20 \\
\hline Pchip & 35.14 & 10.88 \\
\hline Spline & 34.81 & 10.94 \\
\hline
\end{tabular}

\section{Conclusions}

In this paper, different interpolation methods for modelling AC electric arc are presented. Experimental results taken from a steel plant power system and a fluorescence tube are used to show the accuracy of the compared models through computer simulations.

Results have shown that:

- Nearest is fastest in computation time but with a less accuracy;

- Linear requires more computation time than Nearest and the accuracy is a little bit better than that;

- Both PCHIP and Cubic Spline can obtain results with higher accuracy, but for PCHIP the derivative needs to be specified and the derivative of Cubic Spline is not specified but enforced;

- Polynomial interpolation may be good at low orders; however, higher order polynomials may "over-fit" the data and do not predict the curve well in between interpolation points;

- Cubic Spline is much good in smoothly interpolating data.

\section{Acknowledgment}

The paper is financially supported by the Ministry of Science and Technology of Taiwan (R. O. C.), under Grant no. MOST 104-2218-E-194-006.

\section{References}

1. GIGRE Working Group, Electra 36, 5, 35 - 54 (1981).

2. S. Varadan, E.B. Makram, A.A. Girgis, IEEE Trans. Power Deliv. 11, 3, 1685-1691 (1996)

3. M.A.P. Alonso, M.P. Donsion, IEEE Trans. Power Deliv. 19, 1, 367-373 (2004)

4. K. J. Tseng, Y. Wang, D.M. Vilathgamuwa, IEEE Trans. Power Electron. 12, 3, 429-436 (1997) 
5. H. Mokhtari, M. Hejri, Proc. of the IEEE AsiaPacific T \& D Conf. and Exhi., 3, 2078-2083 (2002)

6. G. W. Chang, Y. J. Liu, and C. I. Chen, Proc. of IEEE PES General Meeting, 1-6 (2008)
7. S. C. Wang, C. J. Wu, and Y. J. Wang, International Journal of Circuits, Systems, and Signal Processing, 6, 5, 324-331 (2012)

8. G. D. Gregory, K. Wong, and R. F. Dvorak, IEEE Transactions on Industry Appl., 40, 1006-1011 (2004) 